\title{
Capsule Commentary on Bell et al., Impact of a Randomized Control Educational Trial to Improve Physician Practice Behaviors Around Screening for Inherited Breast Cancer
}

\author{
Joan Neuner, MD MPH \\ Medical College of Wisconsin, Milwaukee, WI, USA. \\ J Gen Intern Med 31(3):326 \\ DOI: $10.1007 / \mathrm{s} 11606-014-3175-4$ \\ (c) Society of General Internal Medicine 2015
}

$\mathrm{T}$ he completion of the human genome project led many to hope that physicians would be making increasing numbers of genetic diagnoses. At the same time, concerns began to develop about the work force needed to provide counseling around those diagnoses. ${ }^{1}$ Bell et al. rigorously studied an intervention to improve the skills of primary care physicians (PCPs) in genetic counseling, and found that that history taking and counseling were incomplete in almost all areas, with many tasks performed by less than $50 \%$ of physicians in either group. There were few differences between intervention groups. ${ }^{2}$

While disappointing, the study's findings suggest several ways forward. The authors compiled an extensive list of 69 tasks important to comprehensive counseling. The list included a number of skills that are needed by physicians who order genetic tests without utilizing a genetic counselor, such as complete history-taking, discussions of test implications, and explorations of ethical and legal issues. This provides a framework for future training and research. The authors suggest that the focus for research should turn to improving genetic counseling referrals. Enhancing electronic medical record (EMR)-based use of risk prediction tools ${ }^{3,4}$ and team-based care could reduce the time required for PCPs to assess risk and select patients for counseling, and could be important ways to improve referrals.
The direction for readying physicians to comprehensively counsel prior to ordering genetic testing is unclear. Primary care scenarios requiring genetic testing have not developed nearly as quickly as some expected, and this may be delaying physicians' acquisition of the skills that are required. The 69 tasks that were compiled show that genetic counselors perform a substantial task, and the 6-hour intervention in this study did not make primary care physicians competent to do the task, even for a familiar scenario. Improving physicians' skills has the potential to better target referrals, and could slow the growth in the need for such counselors. The authors conclude that enhancing the genetic counselor workforce is needed. Team-based care innovations could also help us better partner with genetic counselors. As more genetic tests become available, future research should examine how to develop and distribute the work among such teams.

Corresponding Author: Joan Neuner, MD MPH; Medical College of Wisconsin, Milwaukee, WI 53226, USA (e-mail: jneuner@mcw.edu).

\section{REFERENCES}

1. Moyer VA. Risk assessment, genetic counseling, and genetic testing for BRCA-related cancer in women: U.S. Preventive services task force recommendation statement. Ann Intern Med. 2014;160(4):271-81.

2. Bell RA, McDermott H, Fancher TL, Green MJ, Day FC, Wilkes MS. Impact of a randomized control educational trial to improve physician practice behaviors around screening for inherited breast cancer. J Gen Intern Med. 2014. doi:10.1007/s11606-014-3113-5.

3. Bellcross C. Further development and evaluation of a breast/ovarian cancer genetics referral screening tool. Genet Med. 2010;12(4):240.

4. Ashton-Prolla P, Giacomazzi J, Schmidt AV, et al. Development and validation of a simple questionnaire for the identification of hereditary breast cancer in primary care. BMC Cancer. 2009;9:283. 